\title{
The Gandolfi Stage: A Novel Approach for the Analysis of Single Crystals and Small Volume Samples
}

Gregory Schmidt, XRD Applications Scientist, Thermo Scientific

gregory.schmidt@thermofisher.com

Conventionally, the accurate analysis of single crystals and/or small clusters of crystals requires the use of a single crystal diffractometer. Powder XRD systems are extremely limited in their capabilities for such samples. Capillary spinners are capable of holding such samples, but cannot accurately provide diffraction from more than a few $h \mathrm{kl}$ planes due to the single rotation axis. Other methods for analyzing small volumes on conventional laboratory systems requires the grinding of the sample, but many samples, such as rare minerals, gemstones, newly developed materials consisting of only a few crystals, are not conducive to such alterations., etc.

The Gandolfi Stage, developed for use in conventional powder diffractometers enables the analysis of single crystal or small volume samples without the need of a dedicated single crystal diffractometer. The stage effectively turns the diffractometer into a three-circle system, allowing for the fast analysis of samples previously not suitable for conventional XRD systems. Combining the data collected from this stage, along with various software applications, it is possible to perform a variety of analyses from phase identification to structure solution. In this talk, a variety of analyses of different sample types using the Gandofi stage will be presented. 